\title{
CDISC SDTM Gastrointestinal Test Name Terminology
}

National Cancer Institute

\section{Source}

National Cancer Institute. CDISC SDTM Gastrointestinal Test Name Terminology. NCI Thesaurus. Code C128681.

Terminology associated with the gastrointentestinal test name codelist of the Clinical Data Interchange Standards Consortium (CDISC) Study Data Tabulation Model (SDT M). 